\title{
Drug stability
}

\section{Per Hartvig Honoré}

Hospital pharmacists are experts on the chemical stability of drugs and incompatibilities occurring when different chemical substances are mixed in the same solution. The eminent knowledge gained from basic education in physical, analytical, inorganic and organic chemistry provides a basis, and experience in the hospital environment promotes competence, in different clinical situations. It is because of this reputation that the hospital pharmacy will be asked for help when pharmaceutical problems occur during the handling, preparation and administration of drugs in solution.

Knowledge must be based on scientific reports from experimental studies conducted with adequate planning, protocol design, methods and sound validated analysis. It has been an interest of the European Journal of Hospital Pharmacy science section to publish articles on drug stability and compatibility of a high scientific standard. The basic requirements of a correct stability study with adequate interpretation have already been published in an editorial by our deputy editor in chief, Jean Vigneron from France, in 2008, ${ }^{1}$ and

Correspondence to Professor P Hartvig Honoré, Department of Drug Design and Pharmacology, University of Copenhagen, Universitetsparken 2, Copenhagen 2100, Denmark;

peh@farma.ku.dk this year (2012), a similar contribution from Professor Mike Allwood ${ }^{2}$ from the UK was published, involving good advice on how to perform a study where the results can be published and used in a practical sense in hospital and in the hospital pharmacy.

However, legislation and traditions differ across Europe in terms of which information on stability and incompatibility can be used by healthcare professionals, usually information provided by pharmaceutical companies on their own materials. This information is often very sparse, especially for new drugs. Companies, bound by approved drug information and licensing constraints, are often reluctant to give more information or help in developing additional knowledge. Regulatory authorities look differently on drug information and drug stability and compatibility, leaving most hospital pharmacists in a vacuum, with insufficient information to solve a clinical problem.

We have a number of drug stability databases which can be used to guide clinical practice but the validation of the information may fall short. It is encouraging to see in a previous issue of EJHP a good description of a European working party proposing the generation of stability data to complement regulatory guidelines to fill practical clinical needs. ${ }^{3}$ In all issues of EJHP you will find studies on stability and compatibility, and in this issue there are three more. ${ }^{45}$
Finally, stability and compatibility studies of a high scientific standard are hugely in demand; information is still lacking for many practical situations. EJHP is keen to publish such high standard studies. EJHP seeks to be a leading resource for drug stability information and to support increased knowledge in this area. You are all invited to contribute to this ambitious aim to guide the practising hospital pharmacist.

\section{Competing interests None.}

European Journal of Hospital pharmacy 2012;19:427.

doi:10.1136/ejhpharm-2012-000167

\section{References}

1. Vigneron J. Stability: Ten pieces of advice. EJHP Science 2008;14:2.

2. Allwood M. Assessing the shelf life of aseptically prepared injectabilies in ready to administer containers. EurJ Hosp Pharm 2012;19:277.

3. Bardin C, Astier A, Vulto A, et al. Guidelines for the practical stability studies of anticancer drugs: a European consensus conference. EurJ Hosp Pharm 2012;19:278-85

4. Carati D, Masini C, Minguzzi M, et al. Stability of bortezomib reconstituted under clinical conditions in original vials and polypropylene syringes at $4^{\circ} \mathrm{C}$ and room temperature. EurJ Hosp Pharm 2012;19:428-31

5. Helin-Tanninen M, Autio K, Keski-Rahkonen P, et al. Comparison of six different suspension vehicles in compounding of oral extemporeous nifedipine suspension for paediatric patients. EurJ Hosp Pharm 2012;19:432-37. 